\title{
Electromagnetic Compatibility at the Mains Ports for Medical Equipments
}

\author{
Mircea Ion Buzdugan ${ }^{1}$, Emil Simion ${ }^{1}$, Horia Bălan ${ }^{1}$, Eugen Baru ${ }^{2}$ \\ ${ }^{1}$ Department of Electrical Engineering \\ Technical University from Cluj-Napoca, \\ 15, Constantin Daicoviciu str, Cluj-Napoca (Romania), \\ Phone number: 0040744560 833, Fax number: 0040264410 179, e-mail: mircea.buzdugan@insta.utcluj.ro, \\ horia.balan@eps.utcluj.ro, emil.simion@et.utcluj.ro \\ ${ }^{2}$ Datronix Computer Ltd. \\ 20, Dorului str, Cluj-Napoca (Romania) \\ Phone number: 0040788335665 \\ e-mail: office@datronix.ro
}

\begin{abstract}
The paper presents the retro fitting EMI (electromagnetic interference) filtering measures applied on medical equipments, in this particular case on the four cells galvanic bath ATM-31, produced by Datronix Computer, Romania, in the attempt of achieving electromagnetic compatibility compliance for this product.

Due to the specificity of medical equipments, EMI mains filtering must meet more restrictive safety conditions, as it is presented in the paper, making impossible the use of readymade typical commercial EMI filters.
\end{abstract}

\section{Keywords}

Surge and transient immunity, leakage current, electromagnetic immunity, electromagnetic emissions, Ni Zn ferrites, EMI filters

\section{Introduction}

In order to verify electromagnetic compatibility (EMC) for mains-powered equipments, testing for conducted immunity, according to the EN 61000-4: 2004 standard and conducted emissions, according to the EN 55011: 2007 standard are mandatory.

In the present study of case, these tests were applied to a four cells galvanic bath (figure 1), a therapy equipment which combines the electric current with the thermal effects of the water. The equipment has the capability of generating galvanic or neo faradic currents in its four cells patient-connected.

The supply voltage source of the galvanic bath is a linear one (essentially non-perturbative), without mains EMI filter, but only with a differential mode capacitor $\mathrm{C}_{\mathrm{X}}=100 \mathrm{nF}$ (X2 class) mounted between the $\mathrm{L}$ and $\mathrm{N}$ mains supply conductors (the producer assumed that the inductances of the mains transformer along with the $C_{X}$ capacitor will be enough for the electromagnetic immunization of the equipment to its mains ports). The enclosure of the equipment is on ABS (acrilonytril butadien stiren, a dielectric and thermoplastic material), that is why the equipment is not fitted with PE conductor, being unearthed.

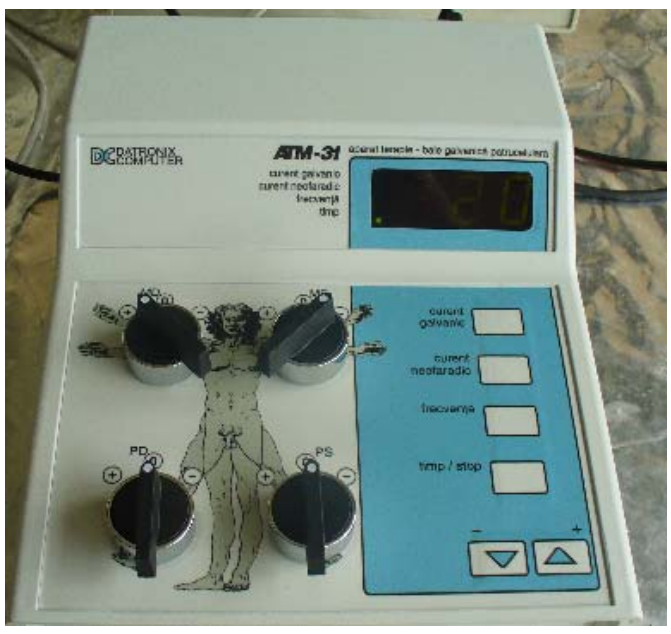

Fig. 1. The galvanic bath ATM-31

The product was ready for the market, and our duty was to verify its EMC compliance and, if necessary, to apply retrofitting immunization measures, in the very restricting safety conditions generally imposed for medical equipments.

Medical equipments must meet the requirements included in the generic standard EN 60601-1: 2006 (Medical electrical equipment. General requirements for basic safety and essential performance). 
According to these prescriptions, a possible risk for electrical shock is present whenever an operator can be exposed to a part at a voltage exceeding $25 \mathrm{~V}_{\mathrm{RMS}}$ or $60 \mathrm{Vdc}$, while an energy risk is present for circuits with residual voltages above $60 \mathrm{~V}$ or residual energy in excess of $2 \mathrm{~mJ}$.

Obviously, the enclosure of the device is the first barrier of protection that can protect the operator or patient from intentional or unintentional contact with these hazards.

Beyond the electrical protection supplied by the enclosure, however, the circuitry of the medical instrument must be designed with other safety barriers to maintain leakage currents within the limits allowed by the safety standards.

Since patient and operator safety must be ensured under both normal and single-fault conditions, regulatory agencies have classified the risks posed by various parts of a medical instrument and have imposed specifications on the isolation barriers to be used between different parts.

The first type of part is the accessible part, a part that can be touched even accidentally (most standards define rigid and articulated probes that must be used to verify the acceptability of enclosure openings).

The second type of part is the live part, a part that when contacted can cause the leakage current to ground or to an accessible part of the equipment to exceed the limits established by the standard. One form of live part is the mains part, defined as a circuit connected directly to the power line.

The third type of part comprises signal-input and signaloutput parts, referring to circuits used to interface a medical instrument to other instruments (for the purposes of displaying, recording, or processing data).

The fourth and most critical part of a medical instrument is that which deliberately comes into physical contact with the patient. Such a part, called an applied part, may include a number of patient connections which provide an electrical pathway between it and the patient.

The patient circuit comprises all patient connections as well as all other parts and circuits of the medical instrument that are not electrically isolated from these connections.

The level of electrical shock protection provided to patients by the isolation of applied parts classifies them as follows:

- Type B: applied parts that provide a direct ground connection to a patient

- Type BF (the F stands for "floating"): indicates that the applied part is isolated from all other parts of the equipment to such a degree that the leakage current flowing through a patient to ground does not exceed the allowable level even when a voltage equal to $110 \%$ of the rated power line voltage is applied directly between the applied part and ground

- Type $C F$ : similar to type $\mathrm{BF}$, but refers to applied parts providing a higher degree of protection, to allow direct connection to the heart

The use of F-type applied parts is preferable in all cases to type $\mathrm{B}$ applied parts. This is because patient environments often involve simultaneous use of multiple electronic instruments connected to the patient. In any case, type B applied parts are prohibited whenever patient connections provide either low-impedance or connections to the patient.

These classifications have more than an academic purpose. The standards provide the designer with clear indications regarding the minimal level of circuit separation and the application of insulation between these parts to accomplish acceptable levels of isolation.

As such, insulation is not only defined as a solid insulating material applied to a circuit, but also as spacings that establish creepage distances and air clearance between parts. The minimum separation distance between elements of two parts is determined by the working voltage between parts as well as by the insulation rating required to afford protection against electrical shock.

A basic insulation barrier is applied to live parts to provide basic protection against electrical shock. Supplementary insulation is an independent insulation barrier applied in addition to basic insulation in order to provide protection against electrical shock in the event of failure of the basic insulation. Double insulation and reinforced insulation provide protection equivalent to the use of both basic and supplementary insulation.

Evidently, the purpose of the various isolation barriers is to ensure that leakage currents are maintained within safe values even when a single-fault condition occurs (ground, enclosure and patient leakage currents).

\section{Immunity testing}

In the specific case of the galvanic bath, the equipment must satisfy to the BF class prescriptions for EMC.

According to the immunity standard EN 61000-4 we have tested the galvanic bath for dips and interruptions, electrostatic discharges, burst and surge, using the Ecompact 4 (Haefely) immunity test equipment.

The equipment passed the dips and interruptions and electrostatic discharges tests, but has failed to the surge $(1.2 / 50 \mu \mathrm{s})$ and to the burst tests. The specific tests carried out with the test setups (figures 2 and 3 ) and with the standard waveforms (figures 4 and 5), were interrupted.

In conclusion, as it was expected, the equipment was not EMC compliant, so we had to improve its conducted immunity on the mains supply network.

The electromagnetic compatibility may often successfully be improved by inserting one or more cells 
of EMI mains filter.

Mains EMI filters carry potentially high currents at dangerously high voltages, so care is essential in their choice. The working voltage and current rating of components can be decided once the specification is known.

The basic specification should also include mechanical details such as the enclosure size, and the limit of weight.

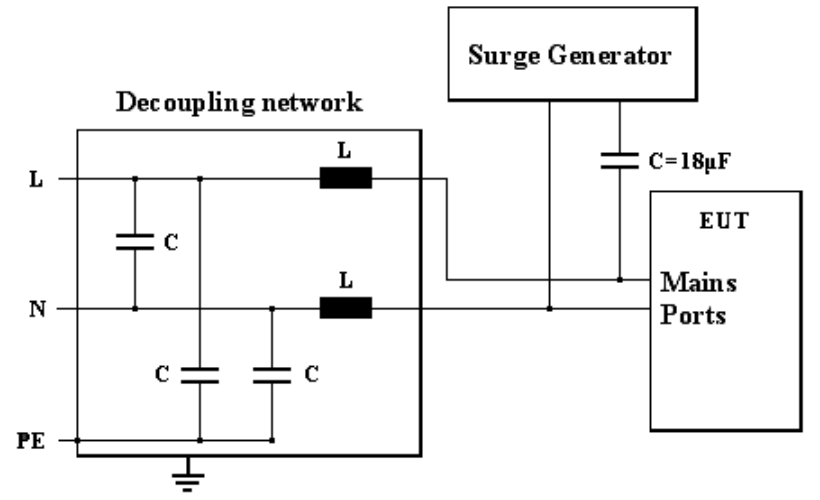

Fig. 2. Surge test setup

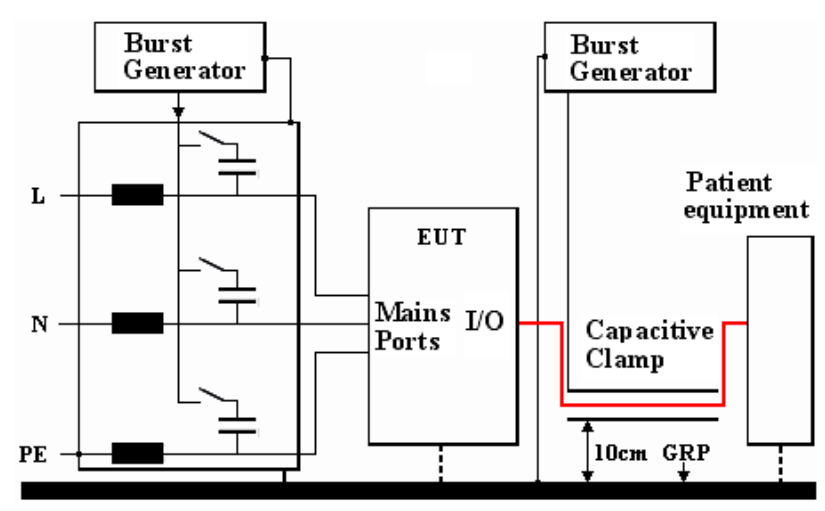

Fig. 3. Burst test setup

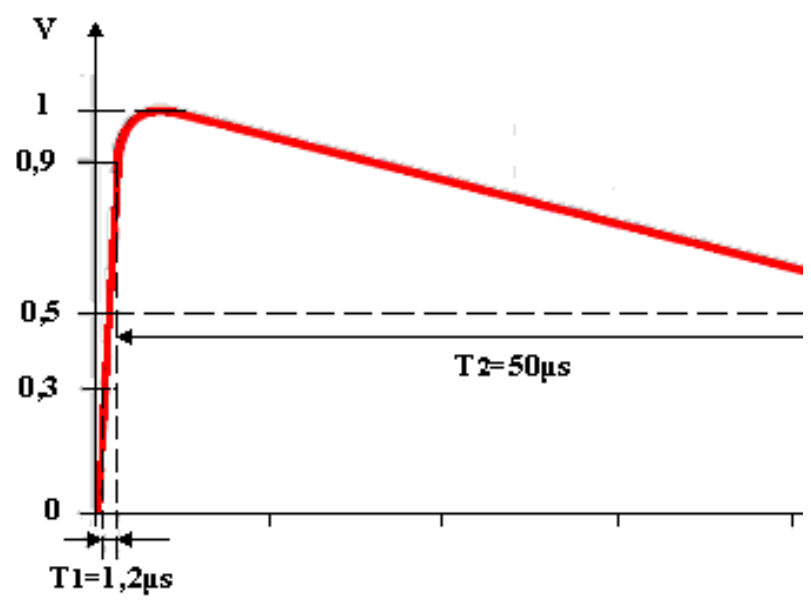

Fig. 4. Surge test waveform

The electrical specification should include the voltage and current rating. In addition the EMC performance and the allowable leakage current should be specified. The electrical specification must also comply with national safety standards.

Filters work on the principal of providing a large discontinuity in the characteristic impedance seen by an unwanted signal. The intention is to reflect most of this unwanted energy back to its source.

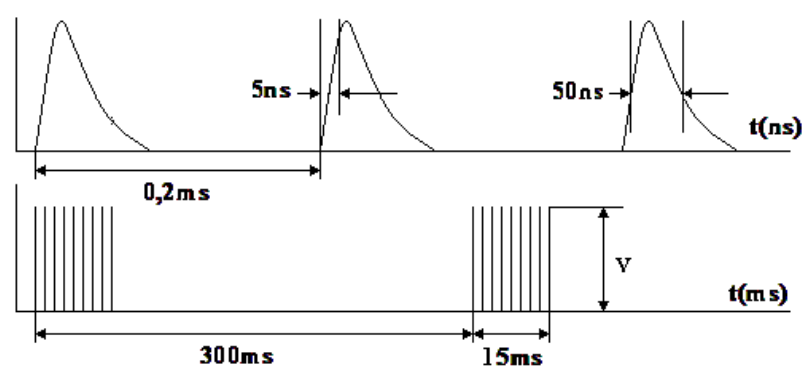

Fig. 5. Burst test waveform

The remaining energy is expended in the inductors through the DC resistance of the coil, the core losses (eddy currents and hysteresis) and the equivalent series resistance of the capacitors. If a filter contains lossy elements, such as a resistor or ferrite component, then the noise energy may be absorbed and dissipated within the filter. If it does not - i.e. if the elements are purely reactive - then the energy is reflected back to its source and must be dissipated elsewhere in the system. This is one of the most important features which distinguish EMI filter design from conventional signal filter design.

Mains filters are tested with a $50 \Omega$ source and load impedance because most RF (radio frequency) test equipments have a characteristic impedance of $50 \Omega$. This allows consistent test results and allows direct comparison between one design and another.

However, because the source and load impedance in practical situations are not generally $50 \Omega$, the attenuation predicted for a design based on this specification is generally optimistic compared with the performance in working equipment.

\section{Counter perturbations measures}

In a filter, the performances of the inductors and capacitors depend critically on the resonant frequencies and on the source and load impedance.

There are two modes of interfering signals. Commonmode signals which means a current that travels along both mains wires in the same direction and returns through earth or ground. Differential signals have a current that travels along one mains wire and returns along the other; thus the sum of the current carried by the two wires is zero, as is the earth current.

The mains power supply is a differential signal with a low frequency. Since the differential mains supply signal carries high current, the filter inductors must be designed so they do not saturate their magnetic cores. 
Most mains filters use common-mode chokes that are wound so that no magnetic flux is produced in the core by a purely differential signal. This is achieved by using an inductor with two windings and arranging for the go and return current to flow through them in opposing directions. Since no magnetic flux is produced, there is no inductive reactance. A common-mode current that flows in the same direction through both supply wires will generate a magnetic flux in the core and will thus have an inductive reactance. The common-mode choke thus appears as having a high series impedance to common-mode signals, but low series impedance to differential signals.

Differential-mode signals are presented with low impedance between the go and return wires by so-called "X capacitors." These X capacitors provide some degree of attenuation to the unwanted signals, but if high levels of attenuation are required, differential-mode chokes may have to be used. Because they must handle mains current these inductors tend to have low values of differential inductance and are physically quite large.

Most mains filters use so-called "Y capacitors" connected between earth and the go and return wires. These Y capacitors typically have values of around a few nanofarads (larger values would exceed earth leakage limits imposed by the relevant safety authorities).

The typical commercial EMI filters are the balanced $\Pi$ type. Although they seem mainly common mode in appearance, they include components to block both common mode and differential mode components. Such a ready-made filter is presented in figure 6.

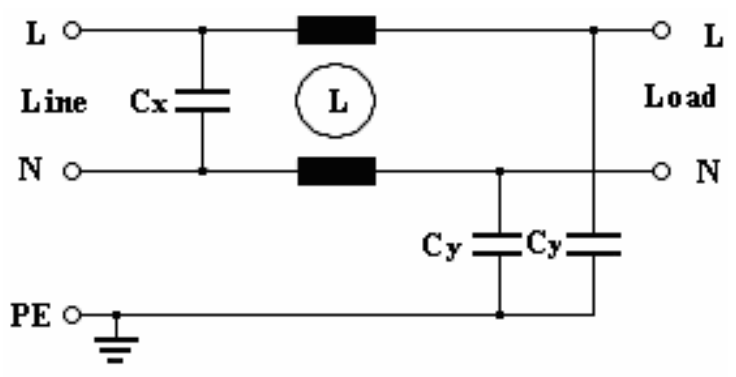

Fig. 6. Typical commercial mains filter

The earth leakage limits imposed on medical equipments, especially if patient-connected, usually makes impossible to use any reasonable size of $Y$ capacitor, because a $50-\mathrm{Hz}$ current of barely $10 \mu \mathrm{A}$ flowing through the heart has the potential of causing ventricular fibrillation and even death.

Instead, such filters have to use better inductors and more filter stages.

To avoid the use of filters, the patient-connected end of the equipment is often made battery-powered and communicates with the mains-powered equipment through an electrically isolated path, such as an optocoupler or fiber optic link.
So, the only possibility for filtering the equipment to the entry stage was a filter configuration like the onepresented in figure 7 , without the $\mathrm{C}_{\mathrm{Y}}$ capacitors.

The varistor was necessary because the galvanic bath was not provided with overvoltage protection and obviously this was the reason for, it failed to the surge test. The very short response time (max. 25ns) of the varistors make them suitable in suppressing overvoltages.

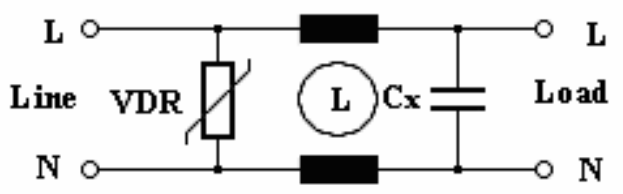

Fig. 7. Typical EMI filter for medical equipments

Because the frequency range of the harmonics of the burst pulses exceed $100 \mathrm{MHz}$, we had to use for the common mode choke a ferrite core, made from $\mathrm{Ni} Z \mathrm{Zn}$ material, the single one capable to handle these frequencies.

The concept of complex magnetic permeability permits the formal separation of the cores impedance into two components:

$\underline{\mu}=\mu^{\prime} \quad j \mu^{\prime \prime}$

- an ideal inductive component (without losses) $X=\omega L_{0} \mu^{\prime}$, where $L_{0}$ represents the air coil inductance (without magnetic core)

- a resistive component, frequency dependent, which quantifies the losses in the material of the magnetic core $R=\omega L_{0} \mu^{\prime \prime}$

We should also note that this formal approach may represent a basic characteristic that allows discriminating efficiently between inductors and ferrites.

Figures 8 and 9 represent the reactive, respectively the resistive part of the impedance of ferrites, which show beyond any doubt that only the $\mathrm{Ni} \mathrm{Zn}$ material is effective for frequencies higher than $100 \mathrm{MHz}$, where the common mode choke becomes dissipative. The other ferrite materials present zero impedance beyond 100 $\mathrm{MHz}$.

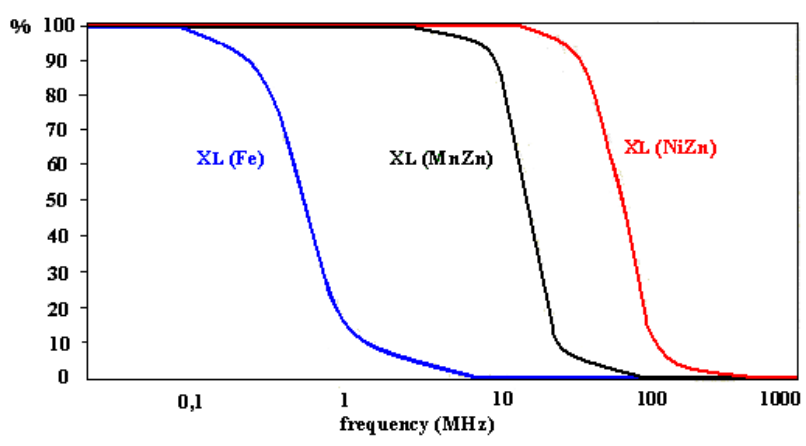

Fig. 8. Reactive component of the ferrite impedance 


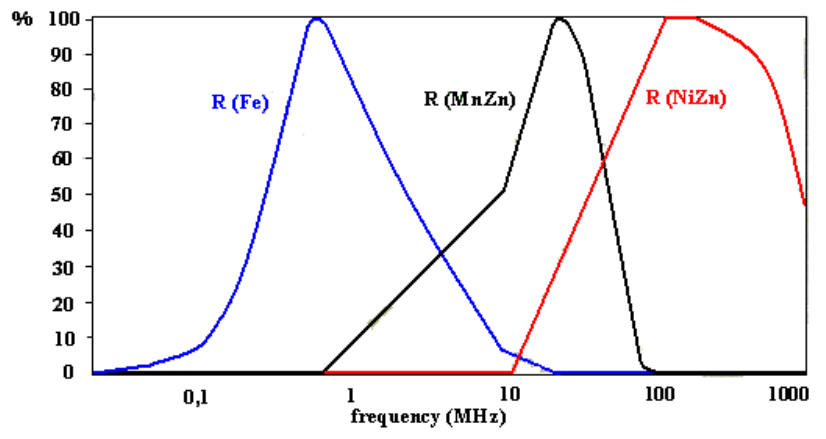

Fig. 9. Resistive component of the ferrite impedance

The filter parameters are:

The selected varistor is JVR $10 \mathrm{~N} 471 \mathrm{~K}$ with the following characteristics:

- response time: $\max .25 \mathrm{~ns}$

- maximum current to 1 surge pulse $8 / 20 \mu \mathrm{s}$ : $2500 \mathrm{~A}$

- maximum current to 2 surge pulse $8 / 20 \mu \mathrm{s}$ : $1250 \mathrm{~A}$

- clamping voltage: $775 \mathrm{~V}$

- typical capacitance at $1 \mathrm{kHz}: 195 \mathrm{pF}$

- maximum operating temperature: $85^{\circ} \mathrm{C}$

It can be seen that the selected varistor is very suitable for suppressing surge pulses.

The $\mathrm{C}_{\mathrm{X}}$ capacitor is MKP $\mathrm{X} 2100 \mathrm{nF} / 250 \mathrm{~V}$ (Epcos) having a rate $\mathrm{dv} / \mathrm{dt}=400 \mathrm{~V} / \mu \mathrm{s}$.
The ferrite has the code 7427010 (WÜRTH ELEKTRONIK) and was wounded with 2.5 turns in common mode winding.

The impedance for one turn is: $Z_{1}=79 \Omega$ at $25 \mathrm{MHz}$ and $\mathrm{Z}_{2}=127 \Omega$ at $100 \mathrm{MHz}$

The impedance for two turns is: $Z_{1}=330 \Omega$ at $25 \mathrm{MHz}$ and $\mathrm{Z}_{2}=513 \Omega$ at $100 \mathrm{MHz}$.

Material: 4W620; $\mathrm{AL}=1300 \mathrm{nH} / \mathrm{N}^{2}$

- $\quad$ operating voltage: $\mathrm{U}_{\mathrm{RMS}}=300 \mathrm{Va} . \mathrm{c}$.

- $\quad$ rated power: $\mathrm{Pn}=0,4 \mathrm{~W}$

- $\quad$ absorbed energy in $2 \mathrm{~ms}: \mathrm{E}=70 \mathrm{~J}$

We have measured the transfer characteristic of the EMI filter using the tracking generator and the spectrum analyzer of the Hameg HM 5014 instrument.

The results are presented in the figure 10 , both in differential (the blue curve with a resonance at $4.5 \mathrm{MHz}$ ) and in common mode (the green curve) in a logarithmic scale of frequency. In common mode, the EMI filter does not present a resonance peak due to the fact that the $\mathrm{C}_{\mathrm{X}}$ capacitor is in short circuit.

In differential mode the EMI mains filter presents a maxim attenuation of more than $55 \mathrm{~dB}$ (a resonance peak), while in common mode the maxim attenuation is only $35 \mathrm{~dB}$ and remains almost the same in a larger range of frequencies. Beyond the frequency of $10 \mathrm{MHz}$ the behaviour of the filter is almost the same both in differential and common mode up to $30 \mathrm{MHz}$.

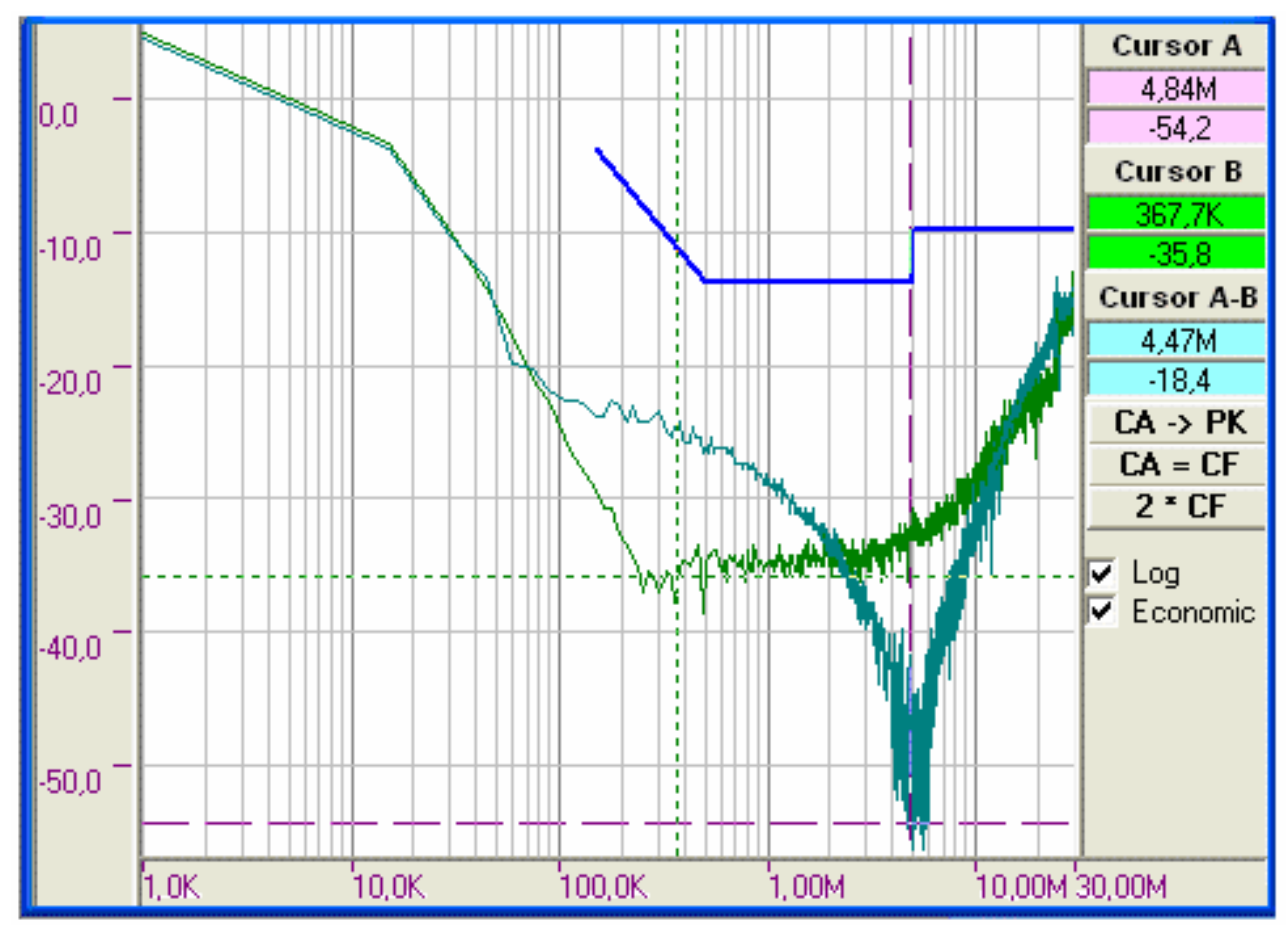

Fig. 10. The transfer characteristics of the designed EMI mains filter in differential (blue) and in common mode (green)

Using the setup presented in figure 11. (spectrum analyzer HM 5014 and line impedance stabilization network HM 6050-2), we have measured the conducted emissions injected by the galvanic bath onto the mains network (the results are presented in the figure 12, in a logarithmic scale of frequency).

We can easily observe that the conducted emissions in instantaneous values do not exceed the standardized average values, according to the EN 50011 standard. 
After repeating the immunity tests of surge (EN 61000-45) and transients (EN 61000-4-2), the equipment passed the tests.

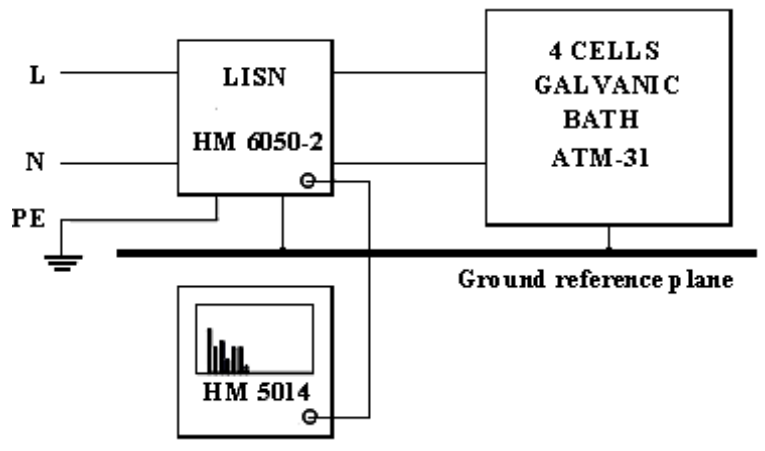

Fig. 11. Test setup for conducted emissions measurement

\section{Conclusion}

Retro fitting counter perturbation measures can improve in some cases, like in the case presented in this paper, electromagnetic compatibility.

In fact, the market for mains filters really took off with the introduction of regulations on conducted mains emissions, compounded by the rising popularity of the switch-mode power supply.

It is important to not consider this approach as a rule in designing products. On a contrary, an EMC product design is the normal way of thinking the development of a product.

EMC design means concern for EMI from the very first step of the design. It means that the designer must

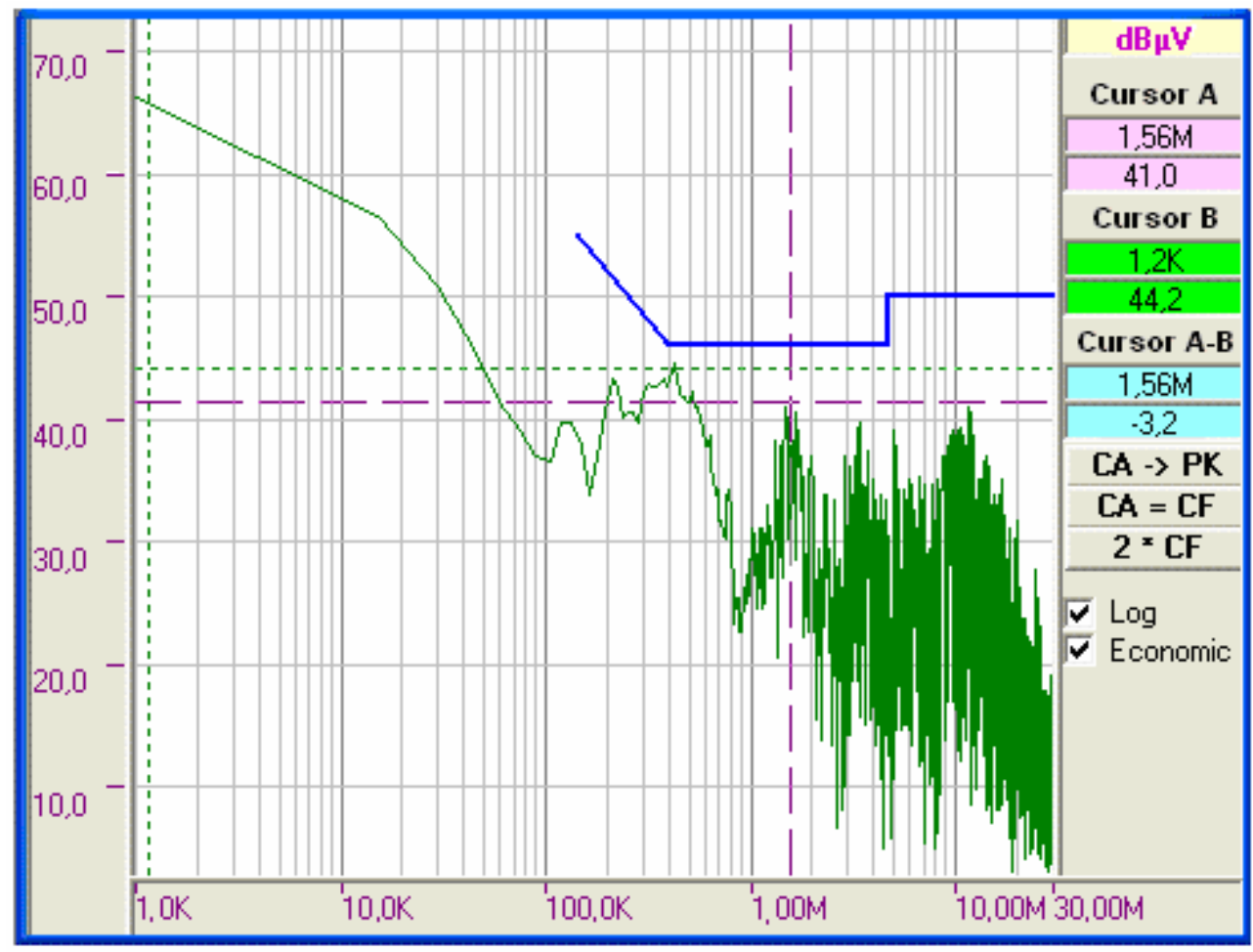

Fig.12. Conducted emissions of the four cells galvanic bath, with the supplementary EMI filter, in a logarithmic scale of frequency

identify the interference sources and the coupling paths, both for conducted and radiated emissions. In the same time the designer must also pay a constant attention to immunity.

\section{References}

[1] Buzdugan, M. I., Electromagnetic Interference at the Mains Ports of an Equipment, ICREPQ `08, Santander, March, 2008

[2] Buzdugan, M. I., Electromagnetic Compatibility-Conducted Interferences, Mediamira Inc., 2008

[3] Williams, T., Using Ferrites for Interference Suppression, Elmac Services, 2004

[4] Gerfer, A., Ralf, B., Zenker,H., Trilogy of Inductors, Swiridoff Verlag, 2002
[5] Ozenbaugh, R. L., EMI Filter Design, Marcel Dekker, Inc., 2001,

[6] Prutchi, D., Norris, M., A Practical Perspective of the Design, Construction and Test of Medical Devices, John Wiley \& Sons, Inc, 2005

[7] Mayergoyz, I. D, Mathematical Models of Hysteresis and Their Applications, Elsevier, 2003

[8] Williams, T., EMC for Product Designers, Newnes, 2007 [9] EN 61000-4: 2004 Electromagnetic Compatibility (EMC) Part 4: Testing and measurement techniques

[10] EN 55011: 2007 Industrial, scientific and medical (ISM) radio-frequency Equipment; Electromagnetic disturbance characteristics; Limits and methods of measurement. 